\title{
Clinical-Community Collaboration: A Strategy to Improve Retention and Outcomes in Low-Income Minority Youth in Family-Based Obesity Treatment
}

\author{
Gina L. Tripicchio, PhD, MSEd,' Alice S. Ammerman, DrPH, 2,3 Dianne S. Ward, EdD, 2,3 \\ Myles S. Faith, PhD, Kimberly P. Truesdale, PhD, Kyle S. Burger, PhD, RD, \\ Kelsey Dean, MS, RD, LD, CCRP, ${ }^{5,6}$ Levent Dumenci, PhD, and Ann Davis, PhD, MPH, ABPP 5,8
}

\section{Abstract}

Background: Clinical-community collaboration is a promising strategy for pediatric obesity treatment, but current research is limited. This study examined the effect of a family-based treatment program embedded in a primary care clinic on retention and changes in child weight status at 1 year.

Methods: Children (2-16 years, BMI $\geq 85$ th percentile, $87.0 \%$ Hispanic) and their parents were recruited from a single pediatric clinic for Healthy Hawks Primary Plus (HHP+). Children were referred by physicians and enrolled by a bilingual clinic-based recruitment coordinator. Participants received 12 weekly 2-hour sessions focused on lifestyle modification and health behavior change and then received bimonthly follow-up visits with their clinic-based physician through 1-year follow-up. Child body mass index (BMI) percentage of the 95 th percentile $\left(\% \mathrm{BMI}_{\mathrm{p} 95}\right)$ was measured as the primary outcome at baseline, postintervention, and 1-year followup. Random effect multilevel models assessed changes in child weight status over time accounting for clustering by family. To further evaluate the impact, HHP+ retention and changes in child weight status were compared to a standard 12-week treatment program only.

Results: HHP+ participants had significantly better retention at 1 year $(73.9 \%, p \leq 0.001)$ compared to the standard treatment program (38.3\%). In HHP+, physician visit attendance was significantly correlated with retention at 1 year $(r=0.69, p \leq 0.001)$, and $\mathrm{HHP}+$ completers had significant reductions in $\% \mathrm{BMI}_{\mathrm{p} 95}$ between baseline and 1-year follow-up $(p=0.03)$.

Conclusion: Clinical-community partnerships might be a promising strategy to improve retention and reduce child weight status in populations currently underrepresented in obesity treatment.

Keywords: behavioral interventions; childhood obesity; health disparities; primary care

\section{Background}

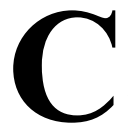
hildhood obesity remains a pressing public health issue. Despite studies indicating that rates are stabilizing in some subgroups, ${ }^{1}$ low-income minority children are disproportionately affected and disparities might be widening in these populations. ${ }^{2}$ Overweight and obesity affect $41.2 \%$ of Hispanic children and $41.8 \%$ of non-Hispanic black children compared to $29.0 \%$ of nonHispanic white children. ${ }^{3}$

While a primary concern is that obesity trends track into adulthood and increase risk for associated comorbidities, the reality is that children with obesity are already experiencing consequences of weight-related disease. ${ }^{4}$ Children

\footnotetext{
'Center for Obesity Research and Education, Temple University, Philadelphia, PA.

${ }^{2}$ Department of Nutrition, Gillings School of Global Public Health, University of North Carolina, Chapel Hill, NC.

${ }^{3}$ Center for Health Promotion and Disease Prevention, University of North Carolina, Chapel Hill, NC.

${ }^{4}$ Department of Counseling, School, and Educational Psychology, University at Buffalo- SUNY, Buffalo, NY.

${ }^{5}$ Center for Children's Healthy Lifestyles \& Nutrition, Kansas City, MO.

${ }^{6}$ Children's Mercy Hospital, Kansas City, MO.

${ }^{7}$ Department of Epidemiology and Biostatistics, Temple University, Philadelphia, PA.

${ }^{8}$ Department of Pediatrics, University of Kansas Medical Center, Kansas City, KS.
} 
with obesity are more likely to develop type 2 diabetes, hypertension, dyslipidemia, sleep apnea, and steatohepatitis $^{5}$ and require immediate intervention.

Currently, multicomponent family-based programs provide the strongest evidence for the treatment of childhood obesity. ${ }^{6,7}$ However, low-income minority populations are underrepresented in these studies, and more data are needed to understand the long-term impact of these programs. ${ }^{8,9}$ One strategy that has been suggested for reaching this population is embedding programs in existing community settings where families currently seek services and support, such as primary care clinics. ${ }^{10}$ This type of integration might assist in reaching low-income families by overcoming barriers to receiving care, improving trust, and involving practitioners who already engage with these populations..$^{11,12}$

Generally, low-income families are less likely to participate in research, ${ }^{13}$ sometimes due to higher levels of stress and lower levels of education. ${ }^{14}$ Parents in these populations are also less likely to recognize child weight issues and, consequently, are less likely to participate in treatment programs. ${ }^{15}$ Programs are often time-intensive and costly to operate, and attrition rates among at-risk populations are high. ${ }^{16,17}$ Therefore, strategies are needed to improve the reach, retention, and impact in populations most in need.

Partnerships between primary care clinics and community programs, including physician program referral, have been suggested as a strategy for more comprehensively addressing pediatric obesity. ${ }^{18,19}$ These partnerships can alleviate the barriers and burdens experienced by medical practitioners attempting to address childhood obesity, including lack of time, training, and resources. ${ }^{20}$ However, evidence on primary care interventions is limited, and few studies have piloted approaches in low-income minority families. ${ }^{21}$ In one study testing a physician referred familybased intervention, children (8-12 years, 54\% black, body mass index $[\mathrm{BMI}] \geq 85$ th percentile) showed significant reductions in BMI z-score (BMIz) at 3 months, but sample size was small $(n=26)$, and no long-term follow-up was measured. ${ }^{22}$ Another study at a pediatric specialty hospital recruited participants through physician referral and tested a 12-month weight management program in low-income children (7-18 years, BMI $\geq 85$ th percentile, $59.7 \%$ Medicaid, $67.3 \%$ black) ${ }^{23}$ While BMIz significantly improved, the study suffered from very high attrition $(81 \%$ loss to follow-up). An additional study recruited low-income Latino children (9-12 years, BMI $\geq 85$ th percentile) to participate in a 6-month family-centered, primary care based program. ${ }^{18}$ Although retention rates were slightly higher in this study $(63 \%)$, there were no significant changes in child weight status at postintervention.

Given the promise and challenges associated with testing family-based programs in a clinical setting, the purpose of this study was to examine the efficacy of a clinicalcommunity partnership to deliver a family-based behavioral group (FBBG) treatment program in a pediatric clinic for a low-income, predominately Hispanic population.
This study is unique in testing the combination of a 12week in-person FBBG program and ongoing physician support from postintervention to 1-year follow-up. In addition, a majority of this study sample is severely obese, and previous trials have identified this as an underrepresented group in similar treatment trials. ${ }^{19}$ It was hypothesized that embedding the program in a clinical setting and engaging physicians would improve child weight status and retention. To further evaluate the impact of $\mathrm{HHP}+$, it was compared to a standard 12-week treatment program only (Healthy Hawks $[\mathrm{HH}]$ ). The primary outcome evaluated changes in child weight trajectory through 1-year follow-up. Secondary outcomes examined participant recruitment, retention, and parent BMI.

\section{Methods}

Healthy Hawks Primary Plus (HHP+) was developed by implementing a standard 12-week FBBG treatment program in partnership with a single pediatric clinic. The program targeted parents and children and was focused on lifestyle and behavior modification strategies to improve physical activity and dietary intake. HHP+ was tailored to be developmentally appropriate based on child age (i.e., children were separated into groups based on age: 2-5, 6-8, 9-12, and 13-18 years) and to support parent language preference (i.e., English and Spanish). Additional details about the 12-week FBBG program $(\mathrm{HH})$ are described briefly below and are available elsewhere. ${ }^{24}$ Before the first session, parents provided informed consent for their children, and if appropriate, children ( $\geq 7$ years) provided assent. All procedures were conducted in accordance with current ethical standards, including those set forth in the Declaration of Helsinki. The Institutional Review Board at the University of Kansas Medical Center reviewed and approved all procedures and protocols.

$\mathrm{HHP}+$ had the following 4 key features that distinguish it from the standard treatment program: $\mathrm{HHP}+(1)$ was operated in partnership with a single pediatric clinic; (2) used a part-time clinic-based recruitment coordinator to manage referrals from physicians, enroll participants, and track participation; (3) trained physicians to deliver bimonthly visits between postintervention and 1-year follow-up; and (4) integrated program participation and behavior modification content into the electronic medical record (EMR) system to support physicians in providing ongoing care.

\section{Clinic-Based Recruitment}

The HHP+ program was initiated in May 2014, and 46 participants were enrolled over 3 cohorts. Participants were enrolled in an ongoing nonrandomized basis, and a new cohort started approximately every 4 months (May 2014, September 2014, and February 2015). Data collection was completed in March 2016. A bilingual program coordinator was hired and trained to enroll participants at the clinic who were referred directly from their physicians. Participants received the 12-week FBBG intervention followed by bimonthly visits with their physicians at the 
clinic. Data were collected at baseline, postintervention, and 1-year follow-up.

\section{Physician Training and Follow-Up Visits}

Physicians and the entire staff at the HHP+ clinic received a 1.5 hour training on 1-2-3-4-5 Fit-tastic! a program designed to promote healthy lifestyles and weight. ${ }^{25}$ The program has five messages that align with the recommended guidelines for the prevention, assessment, and treatment of child overweight and obesity. ${ }^{26}$ The five messages include the following: 1 or more hour of physical activity; 2 hours maximum screen time; 3 servings of lowor nonfat milk or yogurt; 4 servings of water, not sugary drinks; and 5 or more servings of fruits and vegetables. These messages also align with the American Medical Association Expert Committee recommendations for childhood obesity prevention and treatment strategies delivered by physicians as part of routine care. ${ }^{27}$ Physician visits were scheduled by the clinic-based coordinator.

\section{Content Integration in EMRs}

Another feature of HHP+ was the logging of program participation and lifestyle goals in the EMR system. During the FBBG sessions, the coordinator tracked participant attendance and logged participation data into the EMR to keep the physician connected to participant progress. The 1-2-3-4-5 Fit-tastic! goals were also integrated into the EMR system. At the follow-up visits, physicians could reference the information in the EMR and be prompted to provide ongoing support and set new goals.

\section{Standard Treatment Only (Comparison Group)}

To further evaluate the effectiveness of the HHP+ program, the standard treatment $(\mathrm{HH})$ cohorts were used as a comparison group for this study. The $\mathrm{HH}$ intervention was ongoing for almost 10 years and used a multisite, multistrategy recruitment approach to enroll participants. New cohorts began approximately every $4-5$ months, and from April 2006 to February 2014, 300 families in 25 cohorts participated in the $\mathrm{HH}$ program. While both $\mathrm{HH}$ and $\mathrm{HHP}+$ received the 12-week FBBG intervention, HH participants did not receive any intervention contact between postintervention and 1-year follow-up. Although the $\mathrm{HH}$ program has been shown to improve child weight status in the short term, outcomes are modest and attrition is high. ${ }^{24}$ Therefore, this enhanced treatment program (HHP+) was designed to test strategies to improve reach, retention, and outcomes. A diagram showing the intervention components for $\mathrm{HH}$ and HHP+ is presented in Figure 1.

\section{$\mathrm{HHP}+$ Eligibility}

Children had to be $2-18$ years of age, have a BMI greater than or equal to the 85 th percentile, and could not have any other conditions that would prevent them from participating in group-based programs without additional support (e.g., severe Autism Spectrum Disorder). Parents had to be able to speak and write in Spanish or English and be willing to attend sessions and complete measures. In addition, a novel aspect of this study is that all family members were encouraged to attend FBBG sessions and actively participate. If siblings attended sessions and were eligible, they were also enrolled. Inclusion criteria were the same for both HH and HHP+ programs.

\section{Measures}

Baseline measures were administered and collected at the beginning of the first session. Parents self-reported race/ethnicity, date of birth, and gender for themselves and their child. Participants self-identified according to the following race/ethnicity categories: White/Caucasian; Black/African American; Hispanic; and Other. Each variable was dummy coded for inclusion in analyses. Child height and weight were objectively measured at baseline, postintervention ( 3 months), and 1-year follow-up. Trained

Healthy Hawks (HH) Intervention

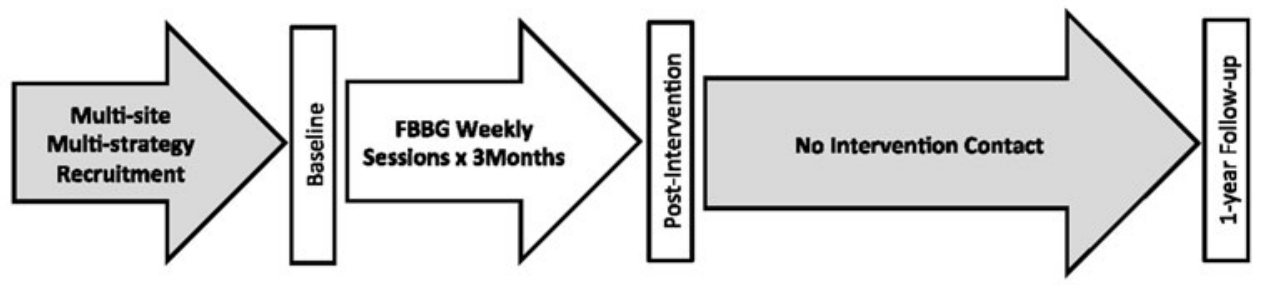

Healthy Hawks-Primary Plus (HHP+) Intervention

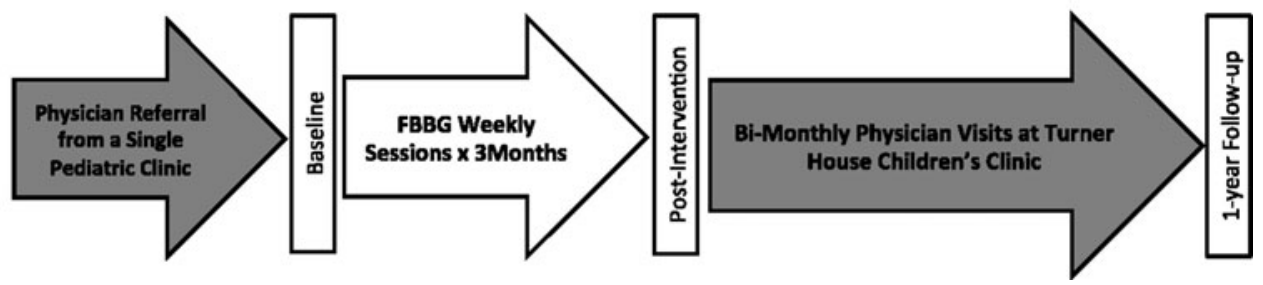

Figure 1. Healthy Hawks (HH) and Healthy Hawks Primary Plus (HHP+) Intervention. 
program staff completed height and weight measures for both parents and children with participants wearing light clothing and no shoes. Height was measured in centimeters (cm) to the nearest $0.1 \mathrm{~cm}$ using a stadiometer (Holtain Ltd., Crymych, Dyfed, United Kingdom), and weight was measured to the nearest 0.01 kilogram $(\mathrm{kg})$ using a digital scale (Temp-Stik Digitron 8000 digital scale National Medical Corp., Temp-Stik Corp). Height and weight were used to calculate children's BMI, and additional variables were derived based on the 2000 CDC growth charts. ${ }^{28}$ Parent height and weight measures were used to derive BMI, and BMI was categorized according to clinical guidelines. ${ }^{29}$

The primary outcome measure for this study was change in child BMI percentage of the 95 th percentile $\left(\% \mathrm{BMI}_{\mathrm{p} 95}\right)$ from baseline to 1-year follow-up. This metric is recommended for capturing change in adiposity in severely obese children. ${ }^{30} \% \mathrm{BMI}_{\mathrm{p} 95}$ is strongly correlated with other child BMI assessments, and a score of 100 is equivalent to the 95th BMI percentile. ${ }^{31}$ A scientific statement from the American Heart Association recommends that $\% \mathrm{BMI}_{\mathrm{p} 95} \geq$ $120 \%$ be used as a measure of severe obesity in children $\geq 2$ years of age, ${ }^{32}$ and other studies suggest that this metric is preferable over other commonly used metrics, such as BMI z-score, especially when evaluating the impact of a obesity treatment program. ${ }^{33} \% \mathrm{BMI}_{\mathrm{p} 95}$ from 100 to 119 is considered moderately obese, and $\% \mathrm{BMI}_{\mathrm{p} 95} \geq 120 \%$ is considered severely obese. ${ }^{34}$

\section{Data Analytic Plan}

Baseline descriptive characteristics were assessed for $\mathrm{HHP}+$ participants, and differences between completers and noncompleters were examined using $t$-tests and chisquared $\left(\chi^{2}\right)$ tests. To evaluate this single arm trial, intentto-treat random-intercept multilevel modeling was used to estimate changes in the child growth curve trajectory over the duration of the intervention and account for clustering by family. A sensitivity analysis was conducted among completers (i.e., participants with completed child weight measures at baseline and 1-year follow-up) using the same analytical approach.

Retention was measured at each time point, and differences in retention were compared between $\mathrm{HH}$ and $\mathrm{HHP}+$ using $\chi^{2}$ tests. In HHP+, the total number of physician visits attended between postintervention and 1-year follow-up was recorded and correlated with participation retention at 1-year follow-up using Pearson's correlations. Finally, random-intercept multilevel modeling was used to compare child growth trajectories between $\mathrm{HH}$ and $\mathrm{HHP}+$ programs from baseline to 1 year. All descriptive analyses were conducted in STATA Version 15, and multilevel random effect models were estimated using Mplus Version 8.

\section{Results}

\section{Participant Characteristics}

Descriptive characteristics for HHP+ and $\mathrm{HH}$ are presented in Table 1. A total of 46 participants were enrolled in the HHP+ program over three cohorts $($ mean $=15.3$ per cohort). HHP+ participants were 9.6 \pm 0.5 years (range $2-16$ years), had a baseline BMI of $28.2 \pm 0.8$, were predominately Hispanic (87.0\%), and Spanish-speaking (76.1\%), 23.9\% female, and $91.1 \%$ received Medicaid. HHP+ parents were $100.0 \%$ female, had a mean BMI of $31.9 \pm 0.8$, and $95.4 \%$ were classified as overweight or obese. There were no differences between HHP+ completers and noncompleters in any variables (age, gender, race/ethnicity, language, insurance type, parent baseline BMI, parent BMI change, child baseline BMI, or severe obesity status at baseline) at postintervention or 1-year follow-up.

\section{Child $\% B M I_{95}$}

Intent-to-treat analysis indicated that $\mathrm{HHP}+\% \mathrm{BMI}_{\mathrm{p} 95}$ decreased between baseline and 1-year follow-up, but not significantly $(\beta=-0.55(0.30), p=0.07)$ (Table 2$)$. HHP+ completers demonstrated significant reductions between baseline and 1-year follow-up ( $\beta=-0.64(0.30), p=0.03)$. When comparing changes in $\% \mathrm{BMI}_{\mathrm{p} 95}$ between $\mathrm{HH}$ and $\mathrm{HHP}+$ from baseline to 1-year follow-up, between-group differences were not significant in intent-to-treat $(\beta=-0.46$ (0.42), $p=0.28)$ or completers analyses $(\beta=-0.51(0.39)$, $p=0.19)$. Mean differences in $\% \mathrm{BMI}_{\mathrm{p} 95}$ among $\mathrm{HHP}+$ and $\mathrm{HH}$ completers at each time point, controlling for family clustering, are visually presented in Figure 2, and results from multilevel models assessing change between $\mathrm{HH}$ and $\mathrm{HHP}+$ from baseline to 1-year follow-up are presented in Table 2 .

\section{Retention}

At postintervention, $76.1 \%$ of HHP+ participants completed follow-up compared to $67.0 \%$ of $\mathrm{HH}$ participants $\left(\chi^{2}=1.52, p=0.22\right)$. At 1 -year follow-up, $73.9 \%$ of HHP+ participants completed follow-up compared to $38.3 \%$ of $\mathrm{HH}$ participants, and these differences were significant $\left(\chi^{2}=20.59, p \leq 0.001\right)$.

\section{Parent BMI}

$\mathrm{HHP}+$ parents did not have significant reductions in BMI at postintervention $(n=33 ; \beta=-0.15(0.15), p=0.30)$ or 1 year $(n=21 ; \beta=0.75(0.72), p=0.31)$.

\section{Physician Visits}

HHP+ participants attended $4.9 \pm 2.3$ physician visits. Physician visit attendance was positively and significantly associated with retention at 1 -year follow-up $(r=0.69$, $p<0.001)$.

\section{Discussion}

Physicians have been identified as important stakeholders and collaborative partners for efforts addressing pediatric obesity. ${ }^{35}$ The current study found that implementing the treatment program in partnership with a pediatric clinic and providing follow-up visits with physicians was feasible and significantly improved participant retention and child $\% \mathrm{BMI}_{\mathrm{p} 95}$ among completers at 1 -year 


\begin{tabular}{|c|c|c|c|}
\hline & $\mathrm{HH}(n=300)$ & HHP+ $(n=46)$ & $p$-value \\
\hline Age (years) & I0.I (0.2) & $9.6(0.5)$ & 0.31 \\
\hline Child BMI & $28.7(0.4)$ & $28.2(0.8)$ & 0.64 \\
\hline \multicolumn{4}{|l|}{ Child \%BMlp95 } \\
\hline Overweight $(<100)$ & $11.0 \%$ & $4.3 \%$ & 0.30 \\
\hline Moderately obese (100-119) & $37.0 \%$ & $34.8 \%$ & \\
\hline Severely obese $(\geq 120)$ & $52.0 \%$ & $60.9 \%$ & \\
\hline Child gender & & & $<0.00 I^{* * *}$ \\
\hline Female & $55.3 \%$ & $23.9 \%$ & \\
\hline Race/ethnicity & & & $0.001 * * *$ \\
\hline White/other & $18.7 \%$ & $2.2 \%$ & \\
\hline Black & $22.3 \%$ & $10.9 \%$ & \\
\hline Hispanic & $59.0 \%$ & $87.0 \%$ & \\
\hline Language & & & $0.003 *$ \\
\hline Spanish & $52.7 \%$ & $76.1 \%$ & \\
\hline Parent gender & & & 0.14 \\
\hline Female & $95.1 \%$ & $100.0 \%$ & \\
\hline Insurance type & & & $0.003 *$ \\
\hline Medicaid & $70.0 \%$ & $91.1 \%$ & \\
\hline Private & $20.7 \%$ & - & \\
\hline None & $9.3 \%$ & $8.9 \%(n=45)$ & \\
\hline Parent BMI & $33.9(0.5)(n=283)$ & $31.9(0.8)(n=43)$ & 0.10 \\
\hline Parent BMI classification & & & 0.08 \\
\hline Normal weight & $8.8 \%$ & $4.7 \%$ & \\
\hline Overweight & $26.9 \%$ & $27.9 \%$ & \\
\hline Obese & $46.6 \%$ & $62.8 \%$ & \\
\hline Severely obese & $17.7 \%(n=283)$ & $4.7 \%(n=43)$ & \\
\hline
\end{tabular}

$* * * p<0.001 ; * p<0.05$.

$\mathrm{HH}$, Healthy Hawks; HHP+, Healthy Hawks Primary Plus; BMI, body mass index; \%BMlp95, body mass index as a percentage of the 95th percentile.

follow-up. This study builds on previous evidence that connecting clinical and community-based care can improve child weight status. ${ }^{36}$

One study in preschool children (predominately white, 25 years) included pediatrician referral and 10 in-person FBBG sessions; significant improvements in weight status were observed for both children and parents at 6 months. ${ }^{37}$ Our study extends these findings to include ethnic minority children 2-16 years of age and tests strategies to enhance patient-physician contact. Another study, which included a diverse population of overweight and obese children 2-12 years (baseline $\mathrm{BMIz}=1.87(0.56), 1$-year $\mathrm{BMIz}$ change $=$ -0.09 ), differed from the present study slightly in intervention delivery (the primary support was delivered by health coaches through bimonthly contacts for 1 year plus twice-weekly text messages or e-mails), but changes in child weight status were similar. ${ }^{19}$

The current study also had a high percentage of severely obese children at baseline $(60.9 \%)$, but we did not observe a significant shift in the number of children who lost enough weight to change categories from severely obese to moderately obese $(n=1)$. Despite an increase in the prevalence of severe obesity and an understanding of the related comorbidities, ${ }^{32,38}$ the current strategies and/or intensity of behavioral lifestyle interventions might not be adequate for supporting treatment of children with severe obesity. ${ }^{39}$ While this study indicates potential by suggesting a solution for high attrition among this population, 


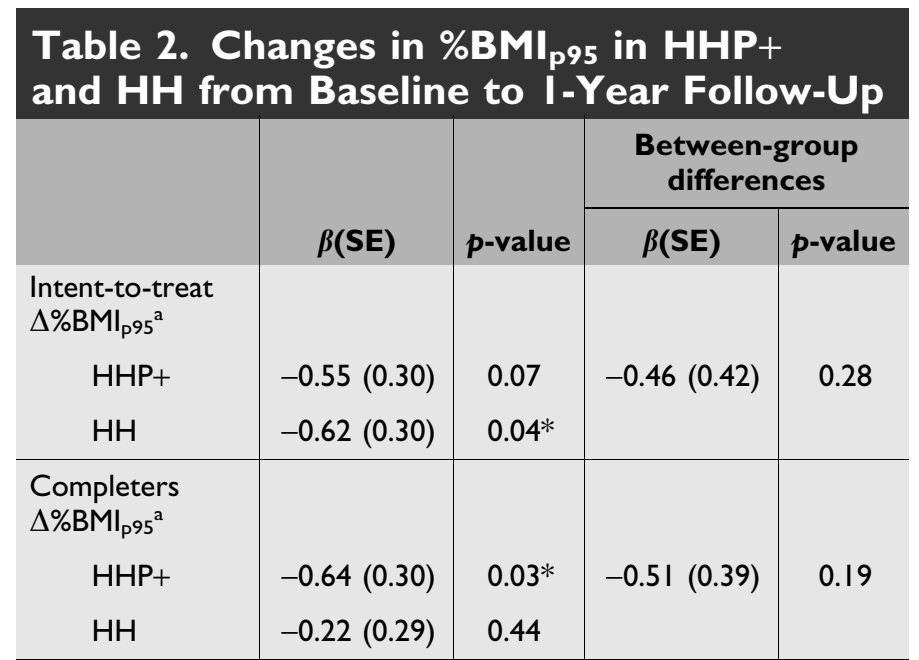

${ }^{a}$ Controlling for clustering by family.

$* p<0.05$.

$\% \mathrm{BMI}_{\mathrm{p} 95}$, body mass index as a percentage of the 95th percentile; $\mathrm{HH}$, Healthy Hawks; HHP+, Healthy Hawks Primary Plus; $\Delta$, change; $\beta$, beta coefficient; $\mathrm{SE}$, standard error; $\mathrm{Cl}$, confidence interval.

Intent-to-treat: $\mathrm{HHP}+n=46, \mathrm{HH} n=300$.

Completers: $\mathrm{HHP}+n=34, \mathrm{HH} n=115$.

approaches should be tested to further elucidate if differential or more intensive treatment approaches are needed for highest risk children. The question of who should be delivering obesity treatment, especially in a clinic-based setting, also warrants investigation.

High attrition rates among Hispanic and African American children in treatment trials present challenges for drawing conclusions and examining long-term impact. ${ }^{40}$ The retention in HHP+ provides promising evidence for much-needed approaches to improve attrition in these groups and, consequently, the ability to identify

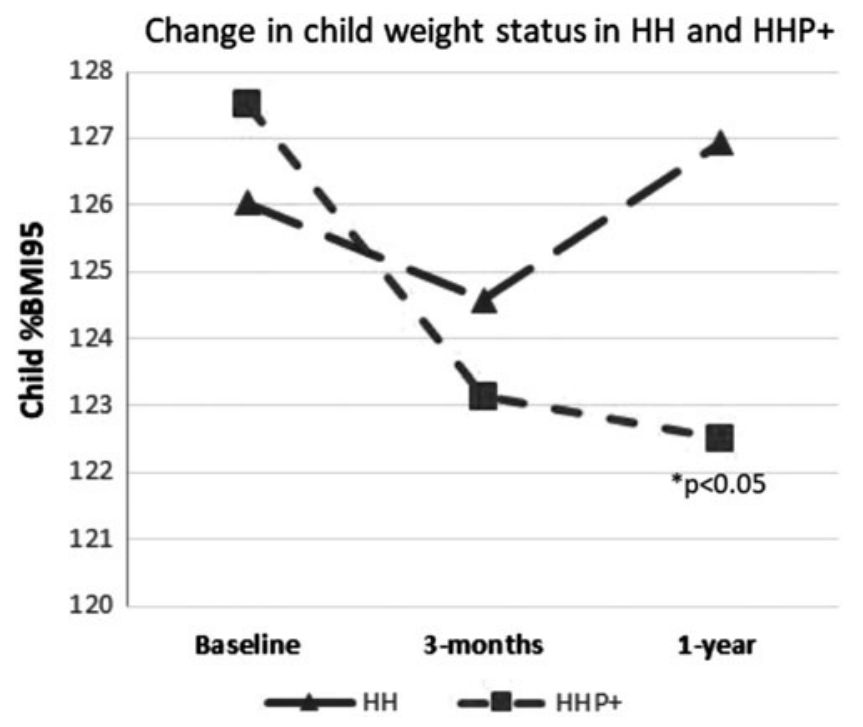

Figure 2. Changes in child \%BMI ${ }_{\mathrm{p} 95}$ among completers at each time point in $\mathrm{HH}$ and $\mathrm{HHP}+$ cohorts. effective treatment strategies. There were 46 participants enrolled in the HHP+ group at baseline and 34 at 1-year follow-up (73.9\%). This rate is significantly better than the standard program, suggesting that ongoing contact with physicians might support long-term retention.

The final follow-up for this intervention was 1 year from baseline, but given the likelihood that children will continue to visit the clinic for regular care, longer term followup should be planned in future studies. It is also relevant to note that the bimonthly physician visits were billed to participants' respective insurance provider and did not require financial support. The study covered $15 \%$ effort for the clinical coordinator to recruit and track participants, and while a rigorous cost-effectiveness evaluation was not conducted, the savings related to recruitment and staff time resulted in overall lower costs for more intervention contact compared to the standard FBBG treatment program. This highlights the potential for dissemination and scalability and offers a strategy for supporting the cost of pediatric obesity treatment programs.

There are various reasons why this approach could have significantly improved participants' retention and shows promise for improving $\% \mathrm{BMI}_{\mathrm{p} 95}$ outcomes. First, using a community-clinic collaboration to increase program credibility has been shown to be an effective retention strategy in other childhood obesity programs in at-risk children. ${ }^{41}$ Increasing overall intervention contact and providing opportunities for one-on-one tailored assistance from physicians could have also contributed to improved retention and reductions in child weight status. ${ }^{42}$ The additional selfmonitoring that occurred as a result of attending the physician visits could have also influenced retention and child $\% \mathrm{BMI}_{\mathrm{p} 95}$ improvements. ${ }^{6,43}$ However, beyond the brief training delivered to physicians and prompts delivered through EMR, we are not sure what was focused on in each session and we do not know the extent to which 1-2-3-4-5 Fit-tastic! was discussed or delivered. We do know that children were weighed at every visit, and parents and children set goals for future success based on personal lifestyle modification targets.

Despite findings to inform future studies, this study has limitations. First, $\mathrm{HH}$ and HHP+ were implemented in a clinical context using nonexperimental designs, making results susceptible to threats to internal validity and limiting the causal inference that can be drawn from the findings. Next, while participants were encouraged to schedule physician visits bimonthly and physicians were trained before the intervention, future studies should more closely monitor visit frequency, content delivered, and visit dose required for meaningful impact. This study, similar to others, only had a modest impact on child weight, and additional information is needed to determine which populations are most appropriate for these programs. In addition, children were not assessed for the presence of comorbidities (e.g., metabolic syndrome, type 2 diabetes, hypertension, and so on). Given the severity of obesity in this population, it is possible that other comorbidities existed, and we were unable to evaluate if participants were experiencing 
additional medical barriers to obesity treatment or if physicians were targeting other health conditions in addition to obesity. Future studies should replicate this approach in larger samples, using more rigorous designs and measures to better assess impact.

This study suggests that initiating a clinic-community partnership to deliver a traditional FBBG program can improve participant retention and child weight status in a population of low-income, predominately Hispanic youth. Hiring a bilingual clinic-based program coordinator, linking program efforts through EMR, and providing postintervention physician visits could also be contributing to the improvements observed. Additional information is needed to determine which populations are best reached with this approach and bolster future child obesity intervention efforts in populations most in need of efficacious treatment.

\section{Acknowledgments}

Funding for this study was provided by the Healthcare Foundation of Greater Kansas City and the Greater Kansas City YMCA. The authors acknowledge the support of our funders, the families and staff who make our programs possible, and Turner House Children's Clinic.

\section{Author Disclosure Statement}

No competing financial interests exist.

\section{References}

1. Olds T, Maher C, Zumin S, et al. Evidence that the prevalence of childhood overweight is plateauing: Data from nine countries. Int $J$ Pediatr Obes 2011;6:342-360.

2. Claire Wang Y, Gortmaker SL, Taveras EM. Trends and racial/ ethnic disparities in severe obesity among US children and adolescents, 1976-2006. Int J Pediatr Obes 2011;6:12-20.

3. Ogden CL, Carroll MD, Kit BK, et al. Prevalence of obesity in the United States, 2009-2010. NCHS Data Brief 2012;82:1-8.

4. Dietz WH. Health consequences of obesity in youth: Childhood predictors of adult disease. Pediatrics 1998;101(3 Pt 2):518-525.

5. Kumar S, Kelly AS. Review of childhood obesity: From epidemiology, etiology, and comorbidities to clinical assessment and treatment. Mayo Clinic Proc 2017;92:251-265.

6. Epstein LH, Valoski A, Wing RR, et al. Ten-year outcomes of behavioral family-based treatment for childhood obesity. Health Psychol 1994;13:373-383.

7. Wilfley DE, Tibbs TL, Van Buren DJ, et al. Lifestyle interventions in the treatment of childhood overweight: A meta-analytic review of randomized controlled trials. Health Psychol 2007;26:521-532.

8. Skelton JA, DeMattia LG, Flores G. A pediatric weight management program for high-risk populations: A preliminary analysis. Obesity 2008;16:1698-1701.

9. Kumanyika SK, Swank M, Stachecki J, et al. Examining the evidence for policy and environmental strategies to prevent childhood obesity in black communities: New direction5s and next steps. Obes Rev 2014;15 Suppl 4:177-203.
10. Kumanyika S, Grier S. Targeting interventions for ethnic minority and low-income populations. Future Child 2006;16:187-207.

11. Chatterjee N, Blakely DE, Barton C. Perspectives on obesity and barriers to control from workers at a community center serving low-income Hispanic children and families. J Community Health Nurs 2005;22:23-36.

12. Minkler M. Community-based research partnerships: Challenges and opportunities. J Urban Health 2005;82(2 Suppl 2):ii3-ii12.

13. Yancey AK, Ortega AN, Kumanyika SK. Effective recruitment and retention of minority research participants. Ann Rev Public Health 2006;27:1-28.

14. Gross D, Julion W, Fogg L. What motivates participation and dropout among low-income urban families of color in a prevention intervention? Fam Relat 2001;50:246-254.

15. Baughcum AE, Chamberlin LA, Deeks CM, et al. Maternal perceptions of overweight preschool children. Pediatrics 2000;106: $1380-1386$.

16. Tershakovec AM, Kuppler K. Ethnicity, insurance type, and follow-up in a pediatric weight management program. Obes Res 2003;11:17-20.

17. Hampl S, Paves H, Laubscher K, et al. Patient engagement and attrition in pediatric obesity clinics and programs: Results and recommendations. Pediatrics 2011;128 Suppl 2:S59-S64.

18. Arauz Boudreau AD, Kurowski DS, Gonzalez WI, et al. Latino families, primary care, and childhood obesity: A randomized controlled trial. Am J Prev Med 2013;44(3 Suppl 3):S247-S257.

19. Taveras EM, Marshall R, Sharifi M, et al. Comparative effectiveness of clinical-community childhood obesity interventions: A randomized clinical trial. JAMA Pediatr 2017;171:e171325.

20. Spivack JG, Swietlik M, Alessandrini E, et al. Primary care providers' knowledge, practices, and perceived barriers to the treatment and prevention of childhood obesity. Obesity 2010;18:1341-1347.

21. Sim LA, Lebow J, Wang Z, et al. Brief primary care obesity interventions: A meta-analysis. Pediatrics 2016;138: pii: e20160149.

22. Pinard CA, Hart MH, Hodgkins Y, et al. Smart choices for healthy families: A pilot study for the treatment of childhood obesity in low-income families. Health Educ Behav 2012;39:433-445.

23. Demeule-Hayes M, Winters MW, Getzoff EA, et al. Pediatric weight management program outcomes in a largely minority, low socioeconomic status population. Clin Med Insights Pediatr 2016; 10:109-114.

24. Davis AM, Daldalian MC, Mayfield CA, et al. Outcomes from an urban pediatric obesity program targeting minority youth: The Healthy Hawks program. Child Obes 2013;9:492-500.

25. Initiative HL. 12345 Fit-Tastic!. Available at http://fittastic.org Last accessed February 25, 2017.

26. Barlow SE, Expert C. Expert committee recommendations regarding the prevention, assessment, and treatment of child and adolescent overweight and obesity: Summary report. Pediatrics 2007;120 Suppl 4:S164-S192.

27. Rao G. Childhood obesity: Highlights of AMA Expert Committee recommendations. Am Family Physician 2008;78:56-63.

28. Ogden CL, Kuczmarski RJ, Flegal KM, et al. Centers for Disease Control and Prevention 2000 growth charts for the United States: Improvements to the 1977 National Center for Health Statistics version. Pediatrics 2002;109:45-60.

29. Clinical guidelines on the identification, evaluation, and treatment of overweight and obesity in adults: Executive summary. Expert panel on the identification, evaluation, and treatment of overweight in adults. Am J Clin Nutr 1998;68:899-917.

30. Flegal KM, Wei R, Ogden CL, et al. Characterizing extreme values of body mass index-for-age by using the 2000 Centers for Disease 
Control and Prevention growth charts. Am J Clin Nutr 2009;90: 1314-1320.

31. Freedman DS, Butte NF, Taveras EM, et al. BMI z-Scores are a poor indicator of adiposity among 2- to 19-year-olds with very high BMIs, NHANES 1999-2000 to 2013-2014. Obesity 2017;25:739-746.

32. Kelly AS, Barlow SE, Rao G, et al. Severe obesity in children and adolescents: Identification, associated health risks, and treatment approaches: a scientific statement from the American Heart Association. Circulation 2013;128:1689-1712.

33. Freedman DS, Butte NF, Taveras EM, et al. The Limitations of Transforming Very High Body Mass Indexes into z-Scores among 8.7 Million 2- to 4-Year-Old Children. J Pediatr 2017;188:50-56.e1.

34. Freedman DS, Berenson GS. Tracking of BMI z scores for severe obesity. Pediatrics 2017;140: e20171072.

35. Faith MS, Stettler N, Pietrobelli A. Engaging primary care clinicians in early obesity prevention research. JAMA 2015;314:823-824.

36. Taveras EM, Marshall R, Kleinman KP, et al. Comparative effectiveness of childhood obesity interventions in pediatric primary care: A cluster-randomized clinical trial. JAMA Pediatr 2015;169:535-542.

37. Quattrin T, Roemmich JN, Paluch R, et al. Efficacy of familybased weight control program for preschool children in primary care. Pediatrics 2012;130:660-666.

38. Lo JC, Chandra M, Sinaiko A, et al. Severe obesity in children: Prevalence, persistence and relation to hypertension. Int $J$ Pediatr Endocrinol 2014;2014:3.
39. Daniels SR, Kelly AS. Pediatric severe obesity: Time to establish serious treatments for a serious disease. Child Obes 2014;10:283-284.

40. Cui Z, Seburg EM, Sherwood NE, et al. Recruitment and retention in obesity prevention and treatment trials targeting minority or low-income children: A review of the clinical trials registration database. Trials 2015;16:564.

41. Buscemi J, Blumstein L, Kong A, et al. Retaining traditionally hard to reach participants: Lessons learned from three childhood obesity studies. Contemp Clin Trials 2015;42:98-104.

42. Force USPST, Barton M. Screening for obesity in children and adolescents: US Preventive Services Task Force recommendation statement. Pediatrics 2010;125:361-367.

43. Michie S, Abraham C, Whittington C, et al. Effective techniques in healthy eating and physical activity interventions: A metaregression. Health Psychol 2009;28:690-701.

Address correspondence to: Gina L. Tripicchio, PhD, MSEd Center for Obesity Research and Education Temple University 3223 North Broad Street, Suite 175 Philadelphia, PA 19140

E-mail: gina.tripicchio@temple.edu 